\title{
SOME PATTERNS IN THE DEVELOPMENT \\ OF CENTERS OF SOLAR ACTIVITY, 1962-66*
}

\author{
Helen W. Dodson and E. Ruth Hedeman \\ (McMath-Hulbert Observatory, The University of Michigan, Pontiac, Mich., U.S.A.)
}

\begin{abstract}
A BST R A C T
A graphical representation of the 66 solar rotations (Carrington) between January 1, 1962 and December 31, 1966 has been prepared. It includes all centers of activity for which the calcium plage attained an area of at least 1000 millionths of the solar hemisphere and/or intensity 3 (McMath scale). In this study the antecedents, descendents, and neighbors of each region can easily be discerned. The work shows clearly that zones of activity, apparently closely related and much larger than single plages existed for long intervals of time. For example, the significant increases in solar activity in February, May, and October of 1965 occurred in a 'family' of calcium plages apparently related through similarities of position and strong radio emission.

The members of 'families' of centers of activity are found at systematically changing longitudes. For some 'families' the change of longitude appears to be primarily a consequence of differential rotation; for others, the pattern of formation of active centers dominates.

According to the data for 1962-66 a meaningful study of the development of a center of activity may require consideration not only of the past history of the zone of the Sun in which it occurs but also of the zone approximately $180^{\circ}$ away on the opposite hemisphere.
\end{abstract}

A graphical representation of the 66 solar rotations (Carrington) between January 1, 1962 and December 31, 1966 has been prepared in an effort to gain insight into the course of solar activity in these years (see Figure 1). This graph includes all centers of activity for which the calcium plage attained an area of at least 1000 millionths of the solar hemisphere or intensity 3 (McMath scale). In this diagram the antecedents, descendents, and neighbors of each region can be discerned easily. Rotations are repeated both horizontally and vertically. It should be borne in mind that Carrington longitudes represent merely a convenient frame of reference corresponding to the mean rotation rate of $27 \cdot 275$ days. Other rotation rates or cadences can be recognized in the display by the lining up of phenomena in non-vertical lines.

The chart of Figure 1 shows that for the years studied zones of activity much larger than single plages existed for long intervals of time, in accord with similar reports by other observers (Becker, 1955; Bezrukava, 1963; Losh, 1939; Martres and Michard, 1965; Mori et al., 1964; Saito, 1964; Vitinsky, 1960) for other time intervals. If this concept is correct, then the study of a single center of activity in 'isolation' represents consideration of only part of a larger and perhaps more fundamental phenomenon.

The chart of plages here presented suggests two types of order or pattern in the data.

* Presented by H.W. Dodson-Prince.

Kiepenheuer (ed.), Structure and Development of Solar Active Regions, 56-63. C I.A.U. 
First there was an apparent concentration in 1962-66 of major activity in two zones, a primary zone with Carrington longitude $\sim 160^{\circ}-230^{\circ}$ and a secondary zone on the opposite side of the Sun at $320^{\circ}-50^{\circ}$. Secondly in these years, apparent families of plages can be traced through the successive returns of a region or by the formation of new regions in neighboring longitudes. Members of these families were often found at systematically changing Carrington longitudes. For some families, the systematic change of longitude was primarily a consequence of differential rotation; for others the pattern of formation of active centers appeared to dominate.

\section{Families of Plages}

The most conspicuous family of plages in the years 1962-66 is the one that appears to be rooted in major solar regions and phenomena in Carrington longitudes near $300^{\circ}$ in January 1962 . This family of plages drifted slowly with time to higher longitudes at rates corresponding to rotation appropriate to very low solar latitudes or to the solar equator itself - a rotation period of $27 \cdot 0$ days or slightly less. It should be noted that this 'family', extending over approximately $70^{\circ}$ of longitude, included regions of both new and old cycle, high and low latitudes, Northern and Southern hemispheres. By the end of 1966 this family had drifted to $\sim 260^{\circ}$ Carrington longitude. In 1962-64 the times of C.M.P. of this family of plages coincided with the times of the principal series of 27-day recurrent geomagnetic storms. In 1965 and early 1966 it included the major centers of activity of the new solar cycle.

The role of differential rotation appropriate to the latitude also can be seen in the recurrence of regions in this presentation. For example, the great March and April regions of 1966 broke out on opposite sides of the Sun in longitudes which can be traced back through differential rotation to the positions of the major regions of May and June 1965, likewise on opposite sides of the Sun. The relationship of the April 1966 region to that of June 1965 can be traced directly through a series of visible plages. For the March 1966 region, one must compute the track of differential rotation in order to establish a possible association with the May 1965 center of activity. This track is shown by a dashed line in Figure 1.

\section{Adjacent Plages}

There are many observations that suggest that the formation and development of a center of activity is not an independent or isolated phenomenon. Not only do plages tend to form in families, but certain active centers appear to beget other centers of activity in their close neighborhoods. This phenomenon is most conspicuous when the general level of solar activity is not too great.

Examples:

(a) On February 5, 1965 an electron-proton flare took place in the old cycle region 


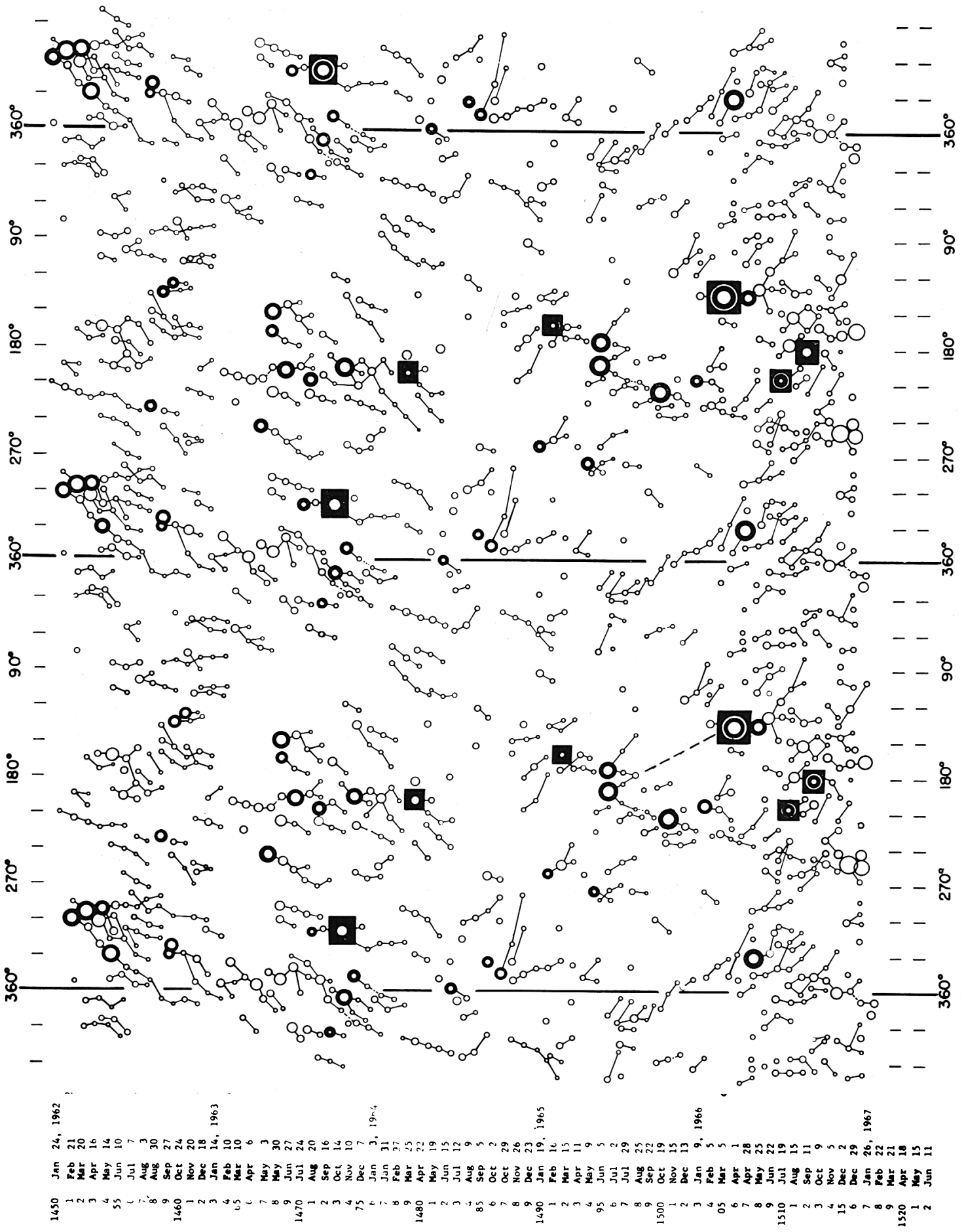


then located at $\mathrm{N} 08^{\circ} \mathrm{W} 25^{\circ}\left(L=160^{\circ}\right)$. In early March, the region returned, diminished in intensity and somewhat fragmented, but bounded on the North by a new cycle region, $\mathrm{N} 20^{\circ}\left(L=170^{\circ}\right)$, and on the South by an old cycle region $\mathrm{S} 04^{\circ}(L=$ $175^{\circ}$ ). (See Figures $2 \mathrm{a}$ and $2 \mathrm{~b}$.)

(b) A center of activity with a $\beta \gamma$ spot formed near the central meridian on December 25, 1965 at the low latitude of $\mathrm{N} 10^{\circ}\left(L=203^{\circ}\right)$. Polarity measurements confirmed its membership in the new cycle. It was the site of three electron-proton flares before West-limb passage. The plage returned in the next rotation diminished in activity, but surrounded by three newly formed regions. (See Figures $2 \mathrm{c}$ and $2 \mathrm{~d}$.)

(c) The formation of the two great flare-rich regions of March and April 1966 in Northern latitudes and on opposite sides of the Sun, was followed during the next two rotations by the development of a band of plages encircling the Northern hemisphere. Figures 3c and 3d show the March region, and the same solar longitudes two rotations later.

Either that which causes the formation of one center of activity, tends to cause numerous centers of activity in the near neighborhood, or the occurrence of a certain type of center of activity in some way sparks the formation of other active centers, perhaps different in kind.

It also can be reported that in 1963-65 new-cycle regions developed in close juxtaposition to major old-cycle regions and in the same longitudes that were and had been the favored zones for old-cycle activity (Dodson and Hedeman, 1967).

\section{Centers of Activity on Opposite Sides of the Sun}

Finally, according to the data for 1962-66 a meaningful study of the development of a center of activity may require consideration not only of the past history of the zone of the Sun in which it occurs, but also of the zone approximately $180^{\circ}$ away on the opposite hemisphere. In 1964-66 there were numerous instances when the formation of a significant center of activity was followed within the course of a rotation by the development of another region on the opposite side of the Sun about $180^{\circ}$ away (Dodson and Hedeman, 1967). Frequently in these years the two regions so placed formed the only significant regions on the sun (see Figure 3 ).

The question immediately arises as to the similarity or difference of regions that occur almost concomitantly on opposite sides of the Sun. According to our studies

Fig. 1. Plot by rotation number and Carrington longitude of all plages with area 1000 millionths of the solar hemisphere and/or intensity 3, 1962-66. The antecedents and descendents of each region are also included. - The size of the circle is an indication of the area of the plage. Heavy dark rings indicate especially flare-rich plages. A dark square outline identifies the regions associated with proton emission and PCA. Time runs from left to right and from top to bottom. Data are repeated for $2 \frac{1}{4}$ rotations. 
(a)
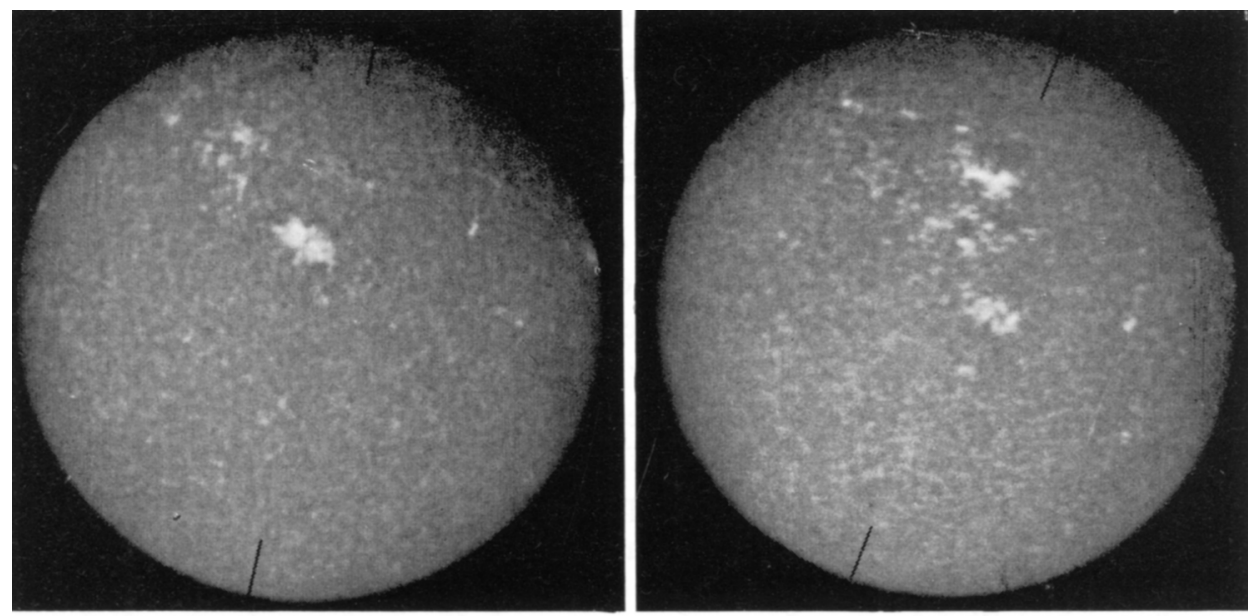

(b)

(c)
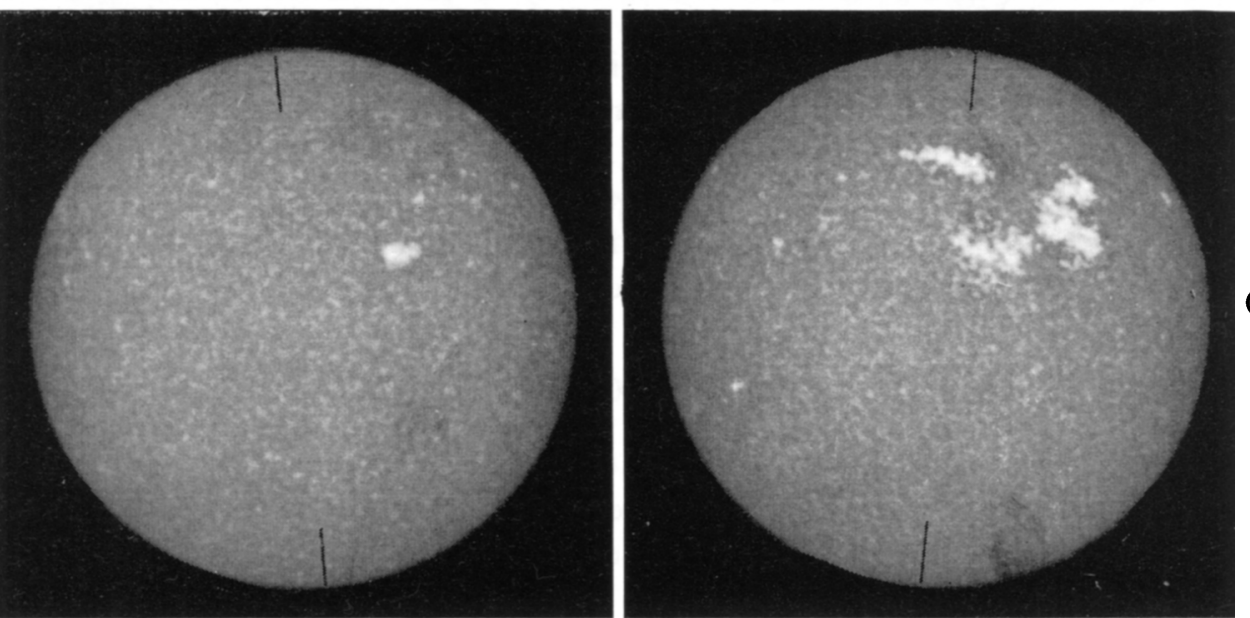

(d)

(e)
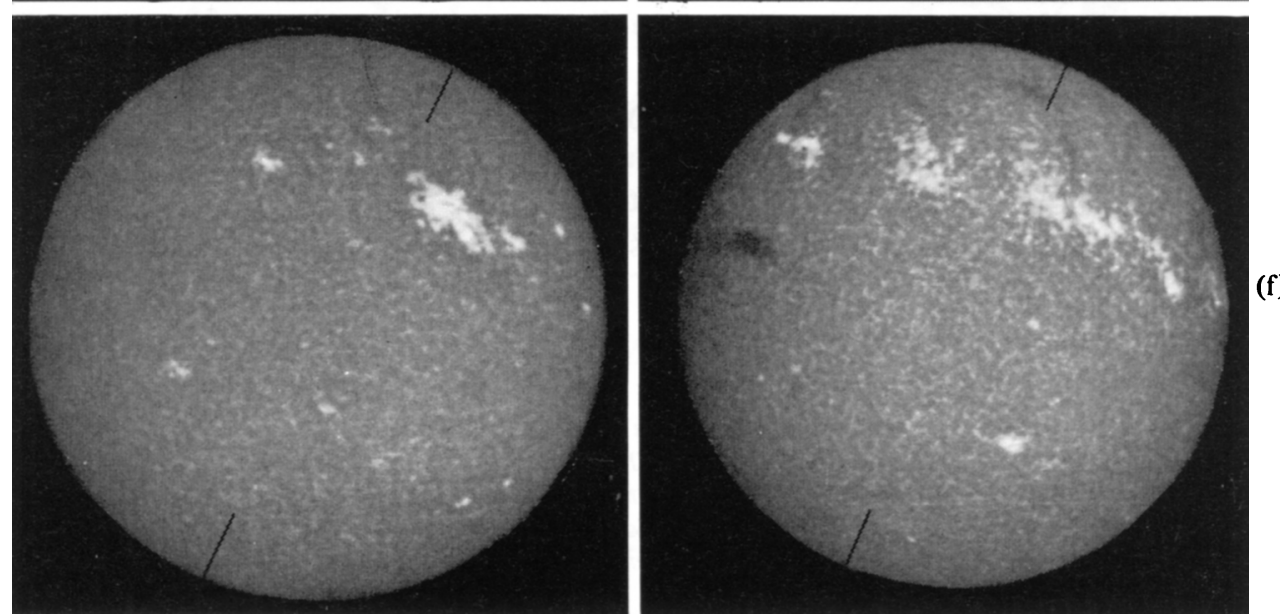

(f) 
the answer to this question is far from clear, but in 1964-66 several of the major cases suggest a possible difference.

In Figure 3a, the region with C.M.P., May 19, 1964 was the most flare-rich region of 1964 . The plage $\sim 180^{\circ}$ away was conspicuous but undistinguished.

The two regions that were near the center of the solar disk on May 21, 1965 (Figure 3c) marked a new high level of solar activity for solar cycle 20 according to both flareand radio-frequency phenomena. The regions opposite them in early and late June were not sufficiently interesting to be' included in the Cooperative Study of Solar Active Regions, May-October 1965.

The March 21, 1966 region (Figure 3e) was the site of numerous proton-electron flares, according to satellite data. Similar particle emission either did not occur with flares in the April 1966 region or such particles did not reach the neighborhood of the Earth.

Finally, the proton-emitting regions of July 7 and August 28 - September 2, 1966 were located near longitude $180^{\circ}$ in late August and early September (Figure $3 \mathrm{~g}$ ). Opposite them, in longitude $354^{\circ}$, there developed in early October a large and conspicuous plage (with only minor spots). In comparison to the other major plages of the second half of 1966 the October region was markedly deficient in radio-frequency emission.

Studies of the gross aspects of centers of activity indicate that there may be recognizable patterns in the development of centers of activity and that there is great need for additional insight into the course of solar activity for the sun as a whole to supplement the fine observations and theoretical interpretation of chromospheric structure and magnetic fields.

\section{References}

Becker, U. (1955) Z. Astrophys., 37, 47.

Bezrukava, A.J.A. (1963) Publications of Pulkovo Obs., 23, 57.

Dodson, Helen W., Hedeman, E.R. (1967) 'The History and Morphology of Solar Activity, 1964-65', in IQSY-COSPAR Symposium, London 1967 (in press).

Losh, H. M. (1939) Publ. Obs. Univ. Michigan, 7, 127.

Martres, M. J., Michard, R. (1965) C.R. Acad. Sc. Paris, 261, 4336.

Mori, S., et al. (1964) Rep. Ionosph. Space Res. Japan, 18, 275.

Saito, T. (1964) Rep. Ionosph. Space Res. Japan, 18, 260.

Vitinsky, J.U.I. (1960) Publications of Pulkovo Obs., 21, 96.

Fig. 2. Calcium spectroheliograms showing the formation of a cluster, or band, of adjacent plages in the rotation following the formation of a center of activity.
a. $1965 \mathrm{Feb} .3\left(\mathrm{~L}=166^{\circ}\right)$
b. 1965 March $2\left(L=172^{\circ}\right)$
c. 1965 Dec. $26\left(L=182^{\circ}\right)$
d. 1966 Jan. $21\left(L=200^{\circ}\right)$
e. $1966 \mathrm{Apr} .4\left(L=319^{\circ}\right)$
f. 1966 May $2\left(L=309^{\circ}\right)$

East is at the left and North at the top of each picture. 
(a)

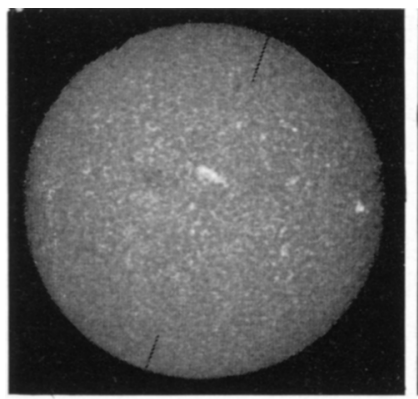

(c)

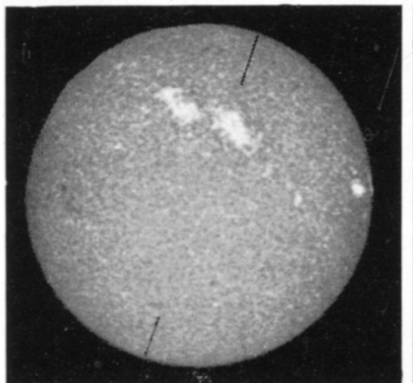

(e)
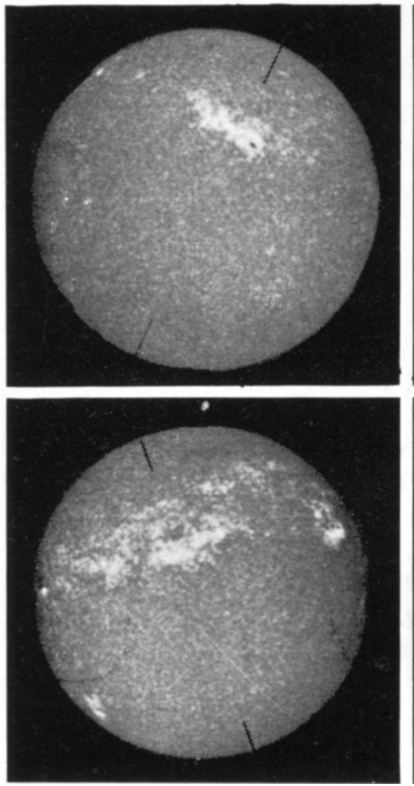

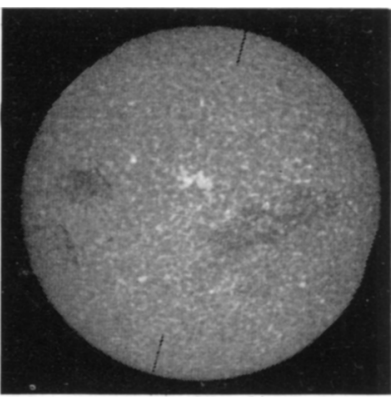

(b)

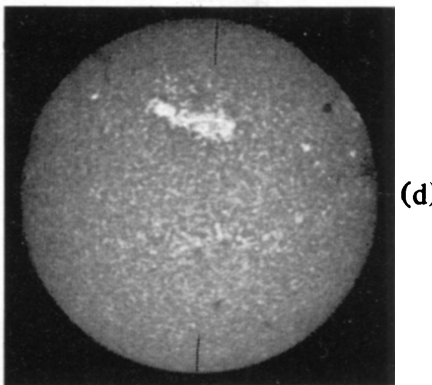

(d)

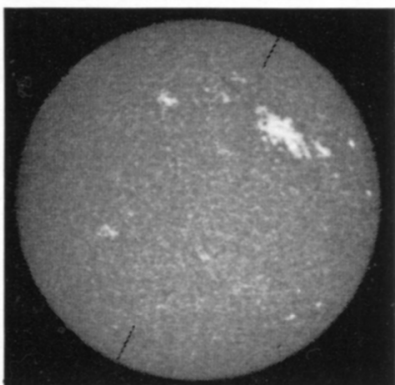

(f)

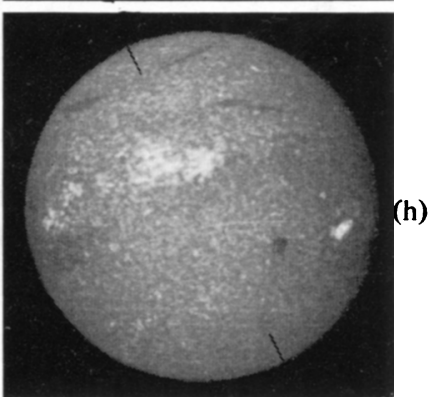

FIG. 3. Calcium spectroheliograms illustrating the formation of centers of activity on opposite sides of the Sun.
a. 1964 May $19\left(L=359^{\circ}\right)$
c. 1965 May $21\left(L=195^{\circ}\right)$
b. 1964 June
$1\left(L=185^{\circ}\right)$
e. 1966 March $21\left(L=144^{\circ}\right)$
d. 1965 June
$30\left(L=26^{\circ}\right)$
g. 1966 Aug. $28\left(L=188^{\circ}\right)$
f. 1966 Apr.
$4\left(L=319^{\circ}\right)$
h. 1966 Oct.
$9\left(L=354^{\circ}\right)$

East is at the left and North is at the top of each picture. 


\section{DISCUSSION}

Bumba: I have some minor comments to your nice talk: Our two complexes of activity observed in 1962 are parts of your two main families of active centers at the beginning of your observational period. The same is true about the situation we showed today at our first slide demonstrating the simultaneous development of active centers of the new and old cycles of activity in the same heliographic longitude.

I saw a manuscript of a paper written by Dr. Berdicevskaja, which will be published in the Soviet Astronomical Journal. Dr. Berdicevskaja estimated the same behaviour of the solar activity distribution during all previous minima of activity, which is possible to study from the Greenwich Photoheliographic Results. Two maxima in the approximate distance of about $180^{\circ}$ may be seen.

Dodson-Prince: I look forward to seeing the interesting manuscript by Dr. Berdicevskaja.

Neupert: We have studied the evolution of the major complex of active regions at longitude $180^{\circ}$ observed in 1962 in conjunction with a study of the extreme ultraviolet emission of these regions as obtained by the satellite OSO-1 during that period. We find that the presence of major activity at this longitude can be traced back through 1960. The relative orientation of three recurring active regions (two in the Northern hemisphere, one in the Southern) is maintained over a period of three years (1960-62).

Dodson-Prince: It is interesting to have our observations in the optical range extended to the extreme ultraviolet, and to earlier years.

Houtgast: I have seen on the slides of Mrs. Dodson some of the $\mathrm{Ca}^{+}$plages surrounded by a dark area and others without such a phenomenon. May I ask for a possible explanation?

Dodson-Prince: The dark feature surrounding certain of the bright plages is the circumfacule identified by Deslandres and D'Azambuja decades ago. It is very conspicuous around some regions but is apparently absent from others. Since the circumfacule is primarily a $\mathbf{K}_{3}$ phenomenon, its absence from spectroheliograms can mean merely an imperfection in instrumental adjustment. It is a solar feature worthy of more study.

Beckers: I observed the circumfacule (the dark region around $\mathrm{K}$-line plages) simultaneously in $\mathrm{H} \alpha$ and $\mathrm{K}$. The circumfacule coincides with the $\mathrm{H} \alpha$ vortex seen around active spot groups. Under good seeing such a vortex is also seen in the $\mathrm{K}$ line. The vortex filament may be the partial or sole cause for the circumfacule. Another cause may be the suppression of the calcium flocculi by the horizontal fields in the vortex.

Sheeley: Circumfaculae occur on $\mathrm{H} \alpha$ core and $\mathrm{K}_{3}$ spectroheliograms taken almost simultaneously (within about $60 \mathrm{sec}$ ). They also occur on $\lambda 8542$ spectroheliograms (taken in the core of the $\lambda 8542 \mathrm{Ca}^{+}$ line), and when the seeing is good these circular dark regions appear striated. In the wings of $\mathrm{Ha}$, say at $+0.7 \AA$, these regions conspicuously lack the small absorbing features that are so characteristic of the $\mathrm{H} \alpha$ wings. A good name for these regions visible on $\mathrm{H} \alpha$ spectroheliograms at $\Delta \lambda=+0.7 \AA$ has been suggested by Beckers, and is 'the runways', where airplanes can land unobstructed by 'the spicule forest'.

Rösch: Trellis has found a correlation between the longitude of new centers and the longitude of Jupiter (based on Greenwich photoheliographic results for many cycles). The appearance of centers at $180^{\circ}$ may be connected to such a type of tidal effect.

Dodson-Prince: It is something to think about. 